\title{
Software Licensing: Pay-Per-Use versus Perpetual
}

Last Revised November 2007

Previous version: May 2006, July 2007.

\author{
Bao-Jun Jiang \\ Pei-yu Chen \\ Tridas Mukhopadhyay \\ baojunj@andrew.cmu.edu \\ pychen@andrew.cmu.edu \\ tridas@andrew.cmu.edu \\ Tepper School of Business \\ Carnegie Mellon University \\ 5000 Forbes Ave
}

Pittsburgh, PA 15213 


\title{
Software Licensing: Pay-Per-Use versus Perpetual
}

\begin{abstract}
Piracy has been a major problem for perpetually licensed software. Usage-based licensing architecture such as pay-per-use or software-as-a-service can offer technology-based protection against piracy. We provide an analytical framework to examine the economic implications of pay-per-use versus perpetual licensing in a market with potential piracy, network effect, and heterogeneous consumers in terms of marginal usage benefit and acquisition costs for pirated software. We show that the potential piracy rate, the user inconvenience cost of pay-per-use licensing, consumer heterogeneity, and the network strength are important factors determining a vendor's optimal choice of licensing architecture. While perpetual licensing tends to be optimal when consumers have homogeneous valuations, pay-peruse is more profitable than perpetual licensing or mixed licensing in markets with heterogeneous consumers and low user inconvenience costs. If the inconvenience cost is low enough, pay-per-use will be more profitable than perpetual licensing even if the market has no potential piracy. The presence of network effect also favors pay-per-use over perpetual licensing; if the network effect is strong, pay-peruse will always dominate perpetual licensing regardless of the inconvenience cost or the potential piracy. With more heterogeneous consumers, higher potential piracy, lower inconvenience costs, and stronger network effects, pay-per-use licensing yields not only higher vendor profits but also a higher social surplus than perpetual licensing. Important managerial implications are also discussed.
\end{abstract}

Keywords: software pricing, piracy, network effect, pay-per-use, usage based pricing, software licensing, perpetual licensing 


\section{Introduction}

Piracy problems have long plagued the software industry. According to the recent Annual Business Software Alliance (BSA) software piracy study, in 2004, expenditures for commercial packaged PC software were $\$ 59$ billion, but software worth over $\$ 90$ billion was actually installed. The 2004 world piracy rate was $35 \%$ and in more than half of the countries studied, the estimated rate was above $60 \%$ (IDC 2005). Fighting piracy is a major challenge for software vendors. Our research explores the software licensing aspect of the piracy problem and examines the usage-based licensing architecture as an effective piracy-prevention mechanism. This paper shows how software makers can utilize a usage-based licensing architecture to increase their market shares and profitability by preventing piracy and by penetrating the light user segment. We will analyze pay-per-use licensing versus perpetual licensing for a software market with heterogeneous consumers, potential piracy, and possible network effects.

Perpetual licensing has been the model commonly adopted by software vendors, especially for PC software. With perpetual licensing, a consumer buys the software and can use it forever. This licensing scheme is also sometimes referred to as "shrink-wrapped licensing" since the full license key is often shipped with the software package itself in a shrink-wrapped box. Such perpetual licensing scheme is prone to software piracy or illegitimate use for several reasons: the software will work forever once the license key is entered, and most often nothing technically prevents the use of the same license key multiple times on different machines or by different users (though the legal license agreement typically limits authorized software use to only one computer or one person). Piracy for software with such licensing mechanisms likely represents the bulk of the revenue leakages due to unauthorized use.

Customer heterogeneity may give rise to another problem with perpetual licensing. Software users may exhibit great heterogeneity; for example, they can be professional users versus novice users, or "casual" (or light) users versus heavy users, or corporate users versus individual consumers. Perpetual licensing is essentially a uniform pricing strategy, which is very likely to be suboptimal in a market with heterogeneous consumers. Differential pricing mechanisms such as creative product bundling and vertical

differentiation (e.g., versioning based on quality levels) have been shown to help vendors realize second- 
degree price discrimination through consumers' self selection into different groups based on their preferences and the product offerings available (Bakos and Brynjolfsson 1999, Shapiro and Varian 1998). Our research examines how usage-based licensing such as pay-per-use can complement or replace perpetual licensing to help independent software vendors deal more optimally with consumer heterogeneity in both marginal usage benefits and pirated software acquisition costs.

Budgetary and productivity pressures resulting from poor or unnecessary software investments prior to the bursting of the "dot-com" bubble may account for the growing current trend toward purchasing decisions focused on how software creates value. Customers increasingly pressure vendors to provide better customer value through more flexible licensing methods. Some firms such as Hyundai are doing more software customization and writing more small software applications themselves, because some software vendors charge by the number of named users or copies installed even though many users use the software only occasionally (Thibodeau 2005). Thus, some customers may find it cheaper to build their own applications offshore than to buy the vendor's software under such perpetual licensing terms.

With perpetual licensing, vendors are essentially targeting only one market segment- - heavy users. The light user segment is left out of the legitimate market because of the high price of perpetual licenses. In most scenarios, perpetual licensing is misaligned with the value creation process on the software user's side. Software vendors need to consider how customer value is created by their software; the value is not created by owning the software but rather by using it. For example, the user value may be based on the number of transactions processed, simulations performed, or reports/files created. Vendors should find out the real drivers of software value and adopt appropriately aligned licensing models. A recent IDC study finds that most vendors and customers strongly believe that the software industry must focus more on clearly establishing software values (Konary et al. 2004). In this paper, we decompose a customer's total software value in terms of its marginal benefit from each use, which may, depending on the situation, be defined as per unit time, per use of a module or feature, or per transaction. This decomposition allows us to better understand users' software valuation and the types of customers drawn by different licensing schemes. 
Usage-based pricing has become increasingly common in both business and consumer software markets. Microsoft Live Meeting Conference Center - a software solution for online meetings, training and events - is currently offered only with pay-per-use and monthly subscription options. ${ }^{1}$ Online software as a service offerings are also examples of usage-based pricing. For example, Salesforce.com's CRM applications are offered only on subscription pricing based on the number of users (at $\$ 10$ to $\$ 65$ per user per month depending on the editions). ${ }^{2}$ CadVantage offers CVSpro-its steel-detailing software - on a pay-per-use basis; customers are charged based on the number of drawings they produce with the software. ${ }^{3}$ Usage-based licensing is also prevalent in the mobile software industry, especially for consumer entertainment software or services. Many software vendors or service providers charge customers based on their actual usage rather than forcing them to purchase perpetual licenses. Flexible licensing and creative pricing has become a critical means for software vendors to achieve broader market penetration and/or deeper account penetration. The success of Agilis Software and Macrovision Corporation in marketing their licensing management solutions demonstrates the value of rapidly customizing product licensing to meet customer needs and to adapt to changes in the market conditions. An IDC (2005) study shows that while most software revenue in the industry is generated from perpetual licensing, $30 \%$ of the software customers believe that the software industry is highly likely to move toward usage-based licensing model as the Internet becomes easier and more cost-effective to access.

Although usage-based licensing schemes are gaining popularity, many software vendors still use perpetual licensing. There is no clear guideline as to what factors are important for choosing the optimal licensing architecture, and the economic implications of usage-based licensing are unclear. Our research helps to fill this gap in the extant literature by addressing the following research questions:

1. What are the benefits and costs of usage-based licensing as compared with perpetual licensing?

2. Under what conditions will the software vendor prefer pay-per-use to perpetual licensing? Will the vendor find it optimal to offer both licensing schemes?

\footnotetext{
${ }^{1} \mathrm{https}: / /$ main.livemeeting.com/ordering/lmbuy it/buy it.cfm\#, last accessed in October, 2007.

2 http://www.salesforce.com/, last accessed in October, 2007.

$3 \underline{\mathrm{http}: / / w w w . s t e e l d e t a i l s . c o m} /$ payperuse.htm, last accessed in October, 2007
} 
3. What are the implications of pay-per-use licensing on the vendor's market share and profit, the consumer surplus and social welfare?

4. How do consumer heterogeneity and the presence of demand-side network effects affect the answers to the previous questions?

We construct an analytical model to examine the economic implications of pay-per-use versus perpetual licensing for a monopolist software vendor facing heterogeneous users in terms of their marginal usage benefit and their acquisition/search cost of pirated software. We find that though perpetual licensing tends to be optimal when consumers have homogeneous marginal benefits, pay-per-use licensing is more profitable than perpetual licensing or mixed licensing when consumers are heterogeneous and the user inconvenience cost of pay-per-use licensing relatively low. In a market with positive network effects, the vendor's optimal perpetual licensing price is an increasing function of both the network strength and the potential piracy rate whereas its optimal profit increases with the network strength but decreases with the piracy rate - a finding that contrasts earlier literature which suggests that in the presence of network effect, higher piracy can lead to higher profits. If the potential piracy is low and the network effect is weak, a vendor offering perpetual licensing may find it optimal to charge a lower price than it would in a market without network effects. This is because, if the network effect is weak, the vendor will find it more profitable to lower its price to increase its customer base than to increase its price to directly reap the benefits of the network effect. Our analyses show that the potential piracy rate, the user inconvenience cost of pay-per-use licensing, consumer heterogeneity, and the network strength are important factors determining a vendor's optimal choice of licensing architecture. We find that if the inconvenience cost is low enough, pay-per-use licensing will be more profitable than perpetual licensing even if the market has no potential piracy. The presence of a positive network effect also favors pay-per-use over perpetual licensing; if the network effect is strong, pay-per-use will always dominate perpetual licensing regardless of the inconvenience cost or the potential piracy. With heterogeneous consumers, higher potential piracy, lower inconvenience costs, and stronger network effects, pay-per-use licensing not only gives the vendor higher profits but also yields a higher social welfare than perpetual licensing. Vendors may find it optimal 
to offer different licensing schemes in isolated markets (e.g., use different licensing mechanisms for software localizations in different countries).

The rest of this paper is organized as follows. Section 2 discusses the related literature. Section 3 describes briefly how the usage-based licensing architecture helps to prevent piracy. Section 4 demonstrates our base model of "utility" software without network effects, and analyzes in turn perpetual licensing, pay-per-use licensing, and mixed licensing. In section 5, we expand our model to analyze a market with positive network effects. Section 6 extends our analysis to general software, which exhibits decreasing marginal usage benefits, and shows that the results are qualitatively the same as those from our analysis of utility software. In section 7 , we discuss the managerial implications and conclude the paper.

\section{Literature Review}

Our research relates to several streams of literature: information goods pricing, software licensing, network externalities, digital rights management (DRM), and software piracy. We extend the existing literature on information goods pricing and software licensing. Sundararajan (2004b) studies optimal pricing strategies for information goods and shows that offering (unlimited usage) fixed fee pricing in addition to a nonlinear usage-based pricing scheme is profit improving and that there may be markets in which a fixed fee pricing is optimal. Essegaier et al. (2002) consider fixed-fee, usage based and two-part tariff pricing for access services in the presence of service capacity constraints; they show that service capacity and consumer usage heterogeneity are two important factors determining a firm's optimal pricing choice. This paper focuses on software products and examines the implications of the architectural differences between usage-based and perpetual licensing; we offer a model that incorporates the salient characteristics of the software market, such as piracy, user heterogeneity, and network effects.

Choudhary et al. (1998) study the economic benefit of renting software, arguing that a monopolist vendor can increase its profit by offering rental software in the first period so as to capture the benefit of higher (delayed) network externalities for its revised version of the software in the second period, because many late-adopters who choose to rent in the first period will make a purchase of the new version in the second period. In a numerical study, Gurnani and Karlapalem (2001) demonstrate that pay-per-use 
licensing can attract casual users and be profitable for a vendor selling packaged software over the Internet. Zhang and Seidmann (2002) demonstrate that in a two-period game, a monopolist vendor can segment consumers based on their sensitivity to product quality and thereby realize second-degree price discrimination through consumers' self-selection behavior. In their model, consumers can either lease the software, which exposes them to the risk of buying into future upgrades with unknown quality, or purchase the software outright, which allows them to make purchasing decisions on future upgrades after the quality of the new version has been determined. Kulatilaka and Lin (2006) study various licensing possibilities (such as fixed fee versus royalty schedules) for innovative technologies. Researchers (e.g., Jiang et al. 2007, Jiang 2007, Ma and Seidmann 2007) have studied other emerging software licensing models such as software as a service (SaaS) and the ad-supported software model in markets with no network effects or piracy. We focus on the economic implications of pay-per-use versus perpetual licensing in a software market with both potential piracy and network effects.

Our research relates to the active research area of digital piracy problems and their solutions. One stream of literature focuses on government instruments (e.g., through copyright enforcement) or technological preventive controls (e.g., encryption or DRM systems) that work to raise consumer piracy costs (e.g., Conner and Rumelt 1991, Shy and Thisse 1999, Chen and Png 2003), while others explore market-based solutions to the piracy problem. Wu and Chen (2005) show that product versioning can be used to fight piracy of information goods and provide a model for product line design in the face of piracy threats. Sundararajan (2004a) investigates a monopolist's optimal pricing schedules and levels of technological protection in a market where the piracy rate is influenced by the level of technological protection and where consumer purchasing decisions are based on prices and the quality differential between legal and pirated goods, which is determined by the vendor's level of DRM implementation. He shows that if a vendor cannot price discriminate, its optimal choice is to implement the maximum level of technology-based protection against piracy; when price discrimination is possible, the vendor will choose to implement a lower level of technological protection. Our research considers usage-based licensing architecture as a technology-based protection against piracy, and offers an economic analysis of software 
licensing architecture in a market with heterogeneous consumers, potential piracy, and demand-side network effects - an approach that, as far as we know, has not been undertaken by other researchers.

August and Tunca (2007) study a software vendor's policy regarding its security patches and find that, when the software security risk is high and the piracy enforcement level is low, or when the potential piracy is high, it is optimal for the vendor to restrict pirated software users from applying security patches. Conner and Rumelt (1991) find that in a market with network externalities, allowing piracy can lead to greater profits than preventing piracy. Their model assumes (rather than derives) an ad-hoc, linear aggregate demand function, whose slope is determined by the efficacy of piracy prevention policies. If only a very small proportion of deterred illegal users will subsequently purchase, firms may prefer to not protect their copyright so that those users can increase the network size and, therefore, justify higher prices to the paying customers. Shy and Thisse (1999) consider software protection policies in a duopoly setting with differentiated products and find that when network effects are strong, both firms will allow piracy at equilibrium. Takeyama (1994) studies a monopolist market with a high valuation group and a low valuation group at the presence of network externalities. Her model assumes that a pirated copy is an imperfect substitute for the original product; its value is thus discounted by a factor. She shows that allowing piracy in such a market can lead to higher firm profits and social welfare than preventing piracy. Essentially, she demonstrates that unauthorized copying can be an efficient way to price discriminate the two types of customers to achieve a large network size; that is, firms "sell" the pirated copies at zero price to the low valuation group while charging higher prices (for the original) to the high valuation group.

Our framework differs from many earlier models in that, rather than assuming an aggregate demand function, we derive demand functions from primitives on consumer preferences. This allows us to closely study how consumers may be affected differently and which segments lose or gain from different types of software licensing. In addition, the earlier models implicitly assume perpetual licensing as the firm's pricing strategy; our paper analyzes perpetual, pay-per-use, and mixed licensing taking into account of multi-dimensional consumer heterogeneity, potential piracy and network effect. We examine the tradeoffs that vendors face when adopting the usage-based licensing architecture. 


\section{Licensing Technology}

We now discuss the differences between the usage-based licensing architecture and the traditional perpetual licensing model. In the latter, a "lifetime" license key is given to consumers who purchase the software. This makes it very convenient for users, who can input the license key once when installing the software and then use the software "forever." Some vendors require users to register within some period of time (otherwise the application will periodically give them annoyances through pop-up reminders). One of the goals of such a registration requirement is to deter the use of pirated software by imposing inconvenience costs on unlicensed users or to foster the perception that vendors possess user registration information to track down and punish illegitimate use. However, the current high rates of software piracy throughout the world provide ample evidence that such perpetual licensing schemes cannot solve the piracy problem, because users can give false registration information, ignore the annoyance features, or simply re-install the software. Compared with usage-based (e.g., pay-per-use) licensing, perpetual licensing is very simple and convenient for customers. This may be why many vendors still use perpetual licensing for many of their software products even though piracy is prevalent.

Usage-based licensing architecture is technologically very different from traditional perpetual licensing. Figure 1 shows conceptually how a usage-based licensing system works. This software licensing architecture, in contrast with perpetual licensing, can essentially eliminate unlicensed software use. The software application itself may be freely distributed over the Internet or via other physical means, but users must establish Internet or other network connections when using the software, to pay for their uses and to activate and validate product features. ${ }^{4}$ After each payment, the user obtains an encrypted code (or a tamper-proof certificate file), which is directly fed into the application. This code encrypts the allowed usage information for each feature definition, as well as information on the expiration time and other relevant attributes. When the software is in use, it automatically and periodically detects any system tampering. The software is disabled if the license has expired, the purchased usage has been consumed, or if any evidence of system tampering is detected. The software

\footnotetext{
${ }^{4}$ Payment can also be made available offline, e.g., through an automated telephone system or monthly billing.
} 
then prompts the user to pay again. In the mobile applications environment, for example, users can pay directly with a credit card, authorize the billing on their monthly mobile bills, or send text messages to a premium number provided by the vendor (Dusparic and Dowling 2003).

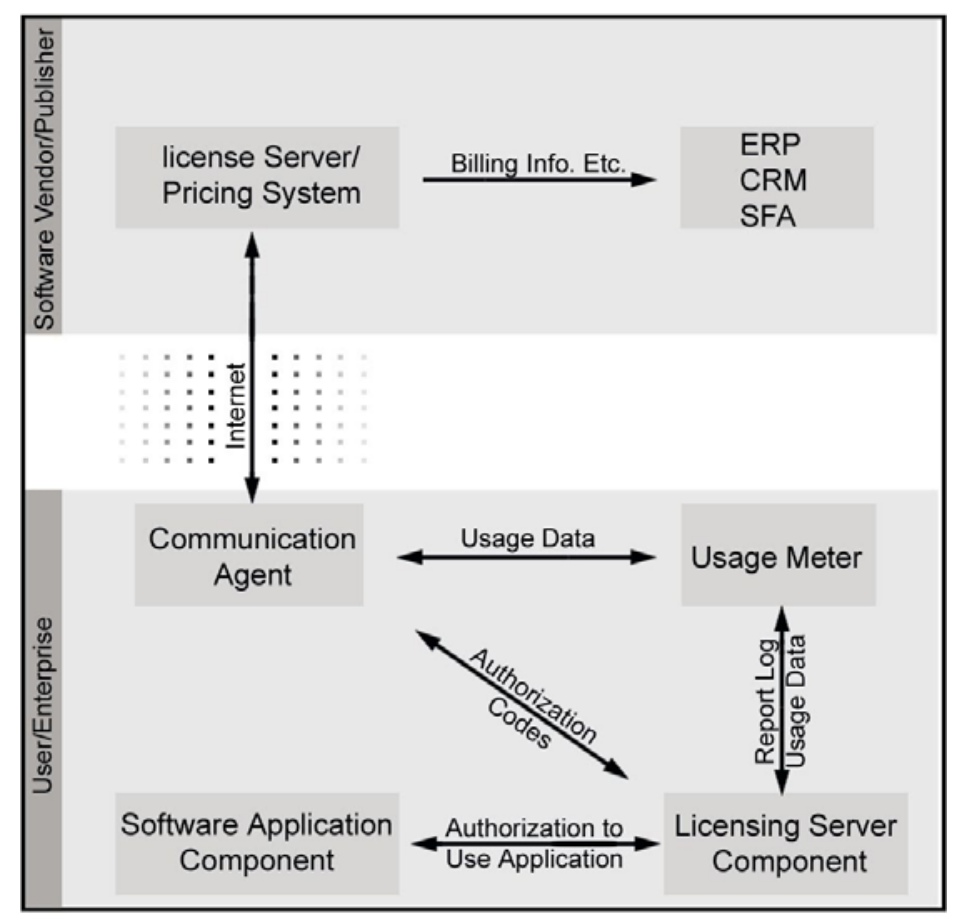

Figure 1: A schematic diagram of a usage-based licensing mechanism ${ }^{5}$

In this paper, we study pay-per-use licensing as a typical example of usage-based licensing. If the vendor sets the maximum allowed usage level per payment low enough and/or configures its software to do online validation frequently enough (say, every 15 minutes), the cost of unauthorized use will become prohibitive relative to its benefit (e.g., 15 minutes may not be enough to finish reinstallation of the software to cheat on usage). This contrasts the case of perpetual licensing, in which users have permanent access to the software once a pirated copy is set up. Such a usage-based licensing architecture makes it virtually impossible or impractical for users to make any unauthorized use of the software. With SaaS applications or online games, for example, a critical component (e.g., dynamic data and core engine) of the software is separated from the client applications distributed to customers, who must pay to gain user

\footnotetext{
${ }^{5}$ The authors thank Hui Chen for help in creating this diagram based on Macrovision product literature.
} 
access to the core component. Hence, if the software is not concurrently offered on perpetual licensing, the usage-based licensing architecture will effectively solve the software piracy problem. Note that perpetual software can also potentially utilize some frequent online validation of licenses. Microsoft has implemented "Windows Genuine Advantage" validation tools for its operating systems, which initially automatically send Microsoft the computer's license data every time the computer is rebooted. After the high pressure (and lawsuits) from users, it reduced the frequency of such validation to once every 90 days. Most legitimate users perceive it unnecessary to validate frequently for such perpetual software. Such validations have clearly not worked even for Microsoft's latest operating systems software (Windows Vista). ${ }^{6}$ While users are not receptive to frequent validations done in perpetual software, they tend to accept the validation in pay-per-use software because they may have to connect to the vendor's servers to access some features or critical and dynamic data. Hence, legitimate users tend to accept some inconvenience in pay-per-use software though they will account for such inconvenience costs when making purchasing decisions. In the following sections, we provide a framework to analyze the economic implications of pay-per-use versus perpetual licensing.

\section{Base Model for Utility Software without Network Effect}

We will first consider what we call "utility" software and analyze a market without any network effect. We will examine network effects in section 5 and general software in section 6 . A user's usage level for utility software varies negligibly when its price changes within a reasonable range. That is, each consumer has a discrete demand for software use - if the price is too high, the consumer will not use the software, but if the price is below a threshold, the user will have a fixed usage level. Operating systems software or anti-virus software, for example, may be considered as utility software. Another example is the software that LASIK surgeons use to profile a patient's cornea; a surgeon's usage level of this software is determined mostly by the number of patients rather than by the price of the software (given

\footnotetext{
${ }^{6}$ Pirated versions of Windows Vista have long appeared on peer-to-peer networks, and are free and fully operational after installation without any validation. To confirm the claim made in many blogs about this, one of the authors removed the purchased Windows Vista from a laptop computer, and then successfully tested a free, pirated version of Windows Vista (downloaded from a peer-to-peer network) without any problem.
} 
that the price satisfies the surgeon's individual rationality constraint). Let $\theta$ denote a customer's expected usage level and $\mu$ denote her marginal benefit per use. We will also refer to $\theta$ as the software usage intensity and $\mu$ as the marginal usage value. For utility software, a user derives a constant marginal usage value ( $\mu$ ) up to her usage level $(\theta)$ and zero marginal value beyond that level. In section 6 , we will model a more general type of software, whose individual marginal usage benefit decreases as a consumer's usage increases; we will show that our main results remain qualitatively the same.

Users may have different usage levels or derive different marginal benefits from the software. For analytical tractability, we assume that consumers' usage levels and marginal usage benefits are both uniformly distributed. Without loss of generality, we normalize both distributions to the unit interval; that is, $\theta \sim$ uniform $[0,1]$ and $\mu \sim$ uniform $[0,1]$.

Per discussion in section 3, perpetual licensing is very susceptible to software piracy. When the vendor uses perpetual licensing, some users may acquire pirated copies rather than buying the software from the vendor. Let $\gamma$ denote a user's total acquisition cost for the pirated perpetual software; $\gamma$ includes the user's search cost and cognitive cost of using pirated software as well as any payment that might be required. Such acquisition cost is assumed to be either high $\left(\gamma_{h}\right)$ or low $\left(\gamma_{l}\right)$. Let $\rho$ denote the proportion of users with low acquisition $\operatorname{costs}\left(\gamma_{l}\right)$ and $P$ denote the price for a perpetual license. Note that no user acquires the pirated software if $P \leq \gamma_{l}$ and that no users will buy the legitimate software if $P \geq \gamma_{h}$. If $\gamma_{l}<P<\gamma_{h}$, the $\gamma_{l}$-type users will use pirated software whereas the $\gamma_{h}$-type will not use pirated software and may potentially buy the licensed software. For expositional ease, we will assume $\gamma_{l}=0$. It is not unreasonable to assume $\gamma_{l}$ to be very close to zero; for example, physical copies of pirated versions of Microsoft's Windows Vista sell for only $\$ 1$ to $\$ 2$ in some markets, and in fact, they are also freely downloadable from some peer-to-peer networks throughout the world. ${ }^{7}$ As a result, the

\footnotetext{
${ }^{7}$ Many news and blog entries about pirated Windows Vista are easily found on the Internet, e.g., http://www.news24.com/News24/Technology/News/0,9294,2-13-1443_2061888,00.html and http://xpress.blogspot.com/2006/12/pirates-crack-windows-vista-in.html, accessed October 2007. In most cases, pirated software may cost nothing in terms of monetary payments because, for example, the pirated versions are
} 
vendor's optimal perpetual license price must clearly satisfy $\gamma_{l}<P^{*}<\gamma_{h}$. In summary, a perpetual software user of type $(\theta, \mu, \gamma)$ has a net utility given by $U(\mu, \theta, \gamma)=\mu \theta-\min [P, \gamma]$.

In light of our discussion in section 3, an inconvenience cost is incurred on pay-per-use users. Let $\delta \in[0,1]$ denote the inconvenience cost per unit of software use; thus, a pay-per-use consumer's net utility is given by $U(\mu, \theta, \gamma)=\left(\mu-P_{\theta}-\delta\right) \theta$, where $P_{\theta}$ is the pay-per-use licensing price (i.e., the price per unit of software use). ${ }^{8}$ If a consumer does not use the software, the utility is zero. We assume that as is typical for information goods, the software vendor's marginal cost is zero and its fixed cost is sunk.

Comparing pay-per-use with perpetual licensing, we prove the following proposition. ${ }^{9}$

Proposition 1: (a) If $\rho>1-1.228(1-\delta)^{2}$, pay-per-use licensing will be more profitable than perpetual licensing; if $\rho<1-1.228(1-\delta)^{2}$, perpetual licensing will be more profitable than pay-per-use.

(b) The vendor's optimal market share is $m=\frac{1-\delta}{2}$ under pay-per-use licensing and $m=0.358(1-\rho)$ under perpetual licensing. Pay-per-use licensing leads to a higher market share if $\delta<0.285+0.715 \rho$.

Proposition 1 implies that when the potential piracy rate and the user inconvenience cost are both relatively high, there is a tradeoff between technological piracy prevention and licensing user convenience. If the potential piracy is high relative to the inconvenience cost of pay-per-use, the vendor should choose the more protective usage-based architecture over the more user-friendly perpetual licensing architecture. A vendor may find it optimal to adopt different licensing architectures in isolated markets. If one country or region has high potential piracy, the vendor should, if possible, offer the payper-use localization of its software in that market rather than one with perpetual licensing. For instance, a

freely available in $p 2 p$ networks. Physical copies of such software, however, may cost $\$ 1$ or $\$ 2$ in certain parts of the world. See footnote 5 about our educational test of acquiring free pirated Windows Vista. But there may still be associated search costs or cognitive costs (e.g., perceived risks of getting sued by the vendor) for some users. ${ }^{8}$ In addition to user inconvenience costs, pay-per-use can incur extra costs on the software vendor, in terms of developing, administering and supporting the more complex licensing system. The parameter $\delta$ can actually capture the effects of such vendor inconvenience cost, if the vendor outsources its licensing management function to a thirdparty solution/service provider on a pay-per-use or revenue-based fee basis. Hence, we can view $\delta$ as the sum of inconvenience costs to both the user and the vendor.

${ }^{9}$ Proof is provided in the Appendix. 
vendor may adopt pay-per-use licensing for software localizations for Asia or Latin America (where $\rho$ is high), while in the US (where $\rho$ is relatively low), it may be profitable to offer perpetual licensing. To capture the benefits of such offerings in the well-connected Internet age, however, the software must have different localizations, otherwise users in the pay-per-use market may be able to acquire pirated software from the perpetual software market.

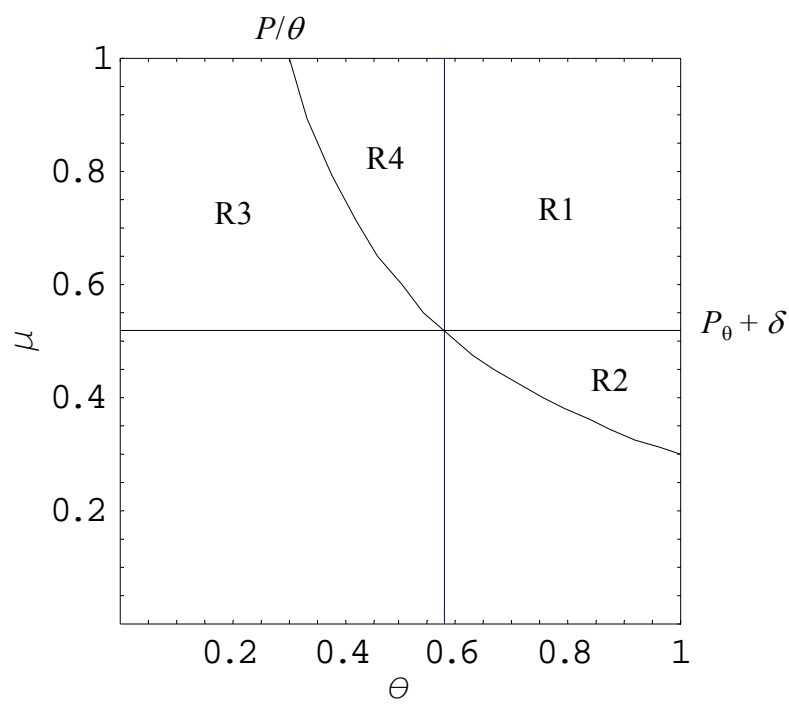

Figure 2: Perpetual vs. pay-per-use licensing.

Proposition 1 indicates that the user inconvenience cost is at least as important a factor as the potential piracy rate in determining the optimal licensing architecture. If the inconvenience cost is low $(\delta<0.097)$, pay-per-use will be more profitable than perpetual licensing even if the potential piracy rate $(\rho)$ is zero. The presence of potential piracy only increases the advantage of pay-per-use over perpetual licensing; that is, as $\rho$ increases, the threshold of $\delta$ below which pay-per-use is more profitable than perpetual licensing will increase. From Propositions 1, it is easy to prove that, regardless of the potential piracy, when pay-per-use is more profitable, the vendor's market share is always larger than what it would be under the optimal perpetual licensing. That is, the optimal pay-per-use licensing allows the vendor to more deeply penetrate the market. The increase in (legitimate) market share has two parts. First, some $\gamma_{l}$ type consumers will become paying customers under pay-per-use whereas they will use pirated software if the vendor offers perpetual licensing. Second, as depicted in Figure 2, by choosing 
pay-per-use over perpetual licensing, the vendor loses some low-valuation, heavy users (region R2), but acquires some high-valuation, light users (in region R3). At the optimal pricing, the vendor gains many more customers than it loses. Note that consumers in regions R1 and R2 are better off under perpetual licensing while consumers in regions R3 and R4 are better off under pay-per-use.

We next consider the possibility that the vendor may offer both licensing schemes. As usual, we assume that fixed costs are sunk. Our model is easily extended to the case in which some or all of the fixed costs are not sunk; the vendor's optimal licensing choice will be straightforwardly determined by comparing the potential profit gain with the incremental fixed costs required. The vendor can choose to offer either or both licensing schemes and then sets the prices. Given the vendor's offering, consumers will choose the option that gives them the highest net utility. Our analysis results in Proposition 2.

Proposition 2: (a) If $\rho$ is relatively low and $\delta$ is relatively high, a monopolist vendor will find it more profitable to offer mixed licensing than either perpetual or pay-per-use licensing alone.

(b) If $\rho$ is relatively high or $\delta$ is relatively low (i.e., $\rho>\delta(2-\delta)$,), the vendor will find it more profitable to offer only pay-per-use rather than either mixed or perpetual licensing.

As illustrated in Figure 2, though adding perpetual licensing to the pay-per-use offering allows the vendor to gain some high-usage, low-valuation customers (region R2), the vendor will lose some profits from the high-usage, high-valuation segment (region R1) because these users will switch from pay-per-use to perpetual licensing, which gives them a lower effective price. In addition, the vendor will lose some $\gamma_{l}$-type pay-per-use customers because they will switch to use the pirated perpetual software. If a large proportion of consumers have high acquisition costs for the pirated software (i.e., the potential piracy is low), adding perpetual licensing to the pay-per-use offering will result in a net gain for the vendor. But if the potential piracy is relatively high (i.e. if $\rho>\delta(2-\delta)$ ), the vendor will find it optimal to adopt only the pay-per-use licensing architecture. When the user inconvenience cost of pay-per-use licensing is negligible, any (positive) potential piracy will make pure pay-per-use licensing optimal. Agilis Software, for example, provides Internet-based licensing solutions to help independent software 
vendors track their customers' usages without imposing high user inconvenience cost, e.g., by automatically logging usage data to the software vendor's hosted Orion Server, or in instances where the users' systems do not have network access providing for the secure logging of the usage data locally, with manual upload of this data to the vendor monthly or quarterly for analysis and billing. ${ }^{10}$ With such reduction in user inconvenience costs, pay-per-use will more likely be the optimal licensing choice.

\section{Software Licensing in a Market with Network Effect}

Since software use often exhibits some network effects, i.e., a consumer's usage benefit may depend on the total number of software users in the market, it is interesting to extend our model to investigate how the presence of network effects will change a vendor's optimal licensing strategy. In particular, we examine whether network effect tends to favor one licensing scheme over the other. For many applications such as word processing software or online video games, as more consumers use the software, a user's benefit from the software is expected to increase. In our framework, the per-use marginal benefit $(\mu)$ can be decomposed into two parts - a random component due to consumer heterogeneity and a non-random component due to network effects: $\mu=\mu_{0}+f(N, \beta)$, where $\mu_{0} \sim$ uniform $[0,1]$, and $f(N, \beta)$ is some non-negative, increasing function of the number of users $(N)$ and the network strength $(\beta)$. Depending on the nature of the software application, $\beta$ can be close to zero (i.e., very weak network effect) or some large positive value (i.e., a very strong network effect). For analytical tractability, we assume that $f(N, \beta)$ takes a linear form: $f(N, \beta)=\beta N$; hence, $\mu=\mu_{0}+\beta N$. We analyze the fulfilled (rational) expectation equilibrium as do many researchers studying network externalities (e.g., Katz and Shapiro, 1985).

If the vendor adopts pay-per-use licensing, all consumers with $\mu_{0}$ that satisfies $\mu_{0}+\beta N-P_{\theta}-\delta \geq 0$ will use the software. Note that $N=1$ if $\beta N-P_{\theta}-\delta \geq 0$ and that $N=1-\left(P_{\theta}+\delta-\beta N\right)$ if $\beta N-P_{\theta}-\delta<0$. Using the fulfilled expectation equilibrium concept, one can easily derive the vendor's optimal prices, market share, and profits,

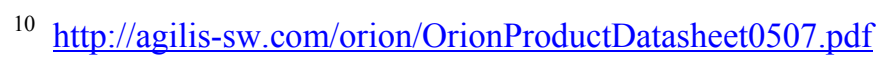


$P_{\theta}^{*}=\left\{\begin{array}{l}\frac{1-\delta}{2}, \text { if } \beta<\frac{1+\delta}{2} \\ \beta-\delta, \text { if } \beta \geq \frac{1+\delta}{2}\end{array}, N^{*}=\left\{\begin{array}{l}\frac{1-\delta}{2(1-\beta)}, \text { if } \beta<\frac{1+\delta}{2} \\ 1, \text { if } \beta \geq \frac{1+\delta}{2}\end{array}\right.\right.$, and $\Pi_{p p u}^{\max }=\left\{\begin{array}{l}\frac{(1-\delta)^{2}}{8(1-\beta)}, \text { if } \beta<\frac{1+\delta}{2} \\ \frac{1}{2}(\beta-\delta), \text { if } \beta \geq \frac{1+\delta}{2}\end{array}\right.$, respectively. The

corresponding consumer surplus and social welfare are given by $C S_{p p u}=\left\{\begin{array}{l}\frac{(1-\delta)^{2}}{16(1-\beta)^{2}}, \text { if } \beta<\frac{1+\delta}{2} \\ \frac{1}{4}, \text { if } \beta \geq \frac{1+\delta}{2}\end{array}\right.$ and $S W_{p p u}=\left\{\begin{array}{l}\frac{(1-\delta)^{2}}{8(1-\beta)}+\frac{(1-\delta)^{2}}{16(1-\beta)^{2}}, \text { if } \beta<\frac{1+\delta}{2} \\ \frac{\beta-\delta}{2}+\frac{1}{4}, \text { if } \beta \geq \frac{1+\delta}{2}\end{array}\right.$, respectively. Proposition 3 follows readily from the above.

Proposition 3: Pure pay-per-use licensing in a market with linear network effect $\mu=\mu_{0}+\beta N$ :

(a) The optimal profit increases with the network strength $(\beta)$, i.e., $\frac{\partial \Pi_{p p u}^{\max }}{\partial \beta}>0$;

(b) If $\beta<\frac{1+\delta}{2}$, the vendor's optimal price is independent of $\beta$ and only consumers with relative highvaluation use the software. If $\beta \geq \frac{1+\delta}{2}$, the optimal price increases linearly with $\beta$ and all consumers will use the software.

As we expect, the presence of network effect increases the vendor's optimal profit. When $\beta$ is below a critical threshold, the vendor's optimal price will be independent of $\beta$. Intuitively, when the network effect is weak, the vendor will find it optimal not to reap the benefit through a price increase but to utilize the network effect to expand its user base. If $\beta$ is high, however, the vendor will cover the entire market at a price that increases linearly with $\beta$. This linear increase is expected since we model a positive linear network effect.

Next we analyze how network effect influences the vendor's optimal price for perpetual licensing. The analysis of perpetual licensing with network effect is much more complex than that of payper-use licensing. In the appendix, we derive the vendor's profit function under rational expectation 
equilibrium. We resort to numerical analysis to determine the optimal prices under the full range of parameter values for $\beta$ and $\rho$; the corresponding profit, consumer surplus and social surplus are subsequently computed. We then compare these values with those under pay-per-use licensing to determine the parameter regions in which each licensing scheme is more optimal (in terms of profit, consumer surplus, or social welfare). We summarize our key results into the following propositions.

Proposition 4: Under perpetual licensing with linear network effect, (a) the vendor's optimal price increases with both $\beta$ and $\rho$ while its optimal profit increases with $\beta$ but decreases with $\rho$. (b) If $\beta$ is below a threshold, there exists some $\rho^{*}>0$ below which the optimal price is lower with network effects than without, and above which the optimal price is higher with network effects than without.

Proposition 5: Pay-per-use licensing becomes more profitable and is more likely to dominate perpetual licensing as the network strength increases. If the network strength is large enough $(\beta>1.8)$, pay-peruse will always yield a higher profit than perpetual licensing for any piracy rate or inconvenience cost. Proposition 6: If $\delta$ is relatively low (or if $\rho$ is relatively high), pay-per-use licensing yields a higher consumer surplus and social welfare than perpetual licensing. In addition, as the network strength increases, pay-per-use will result in more favorable consumer surplus and social welfare than perpetual licensing. If $\beta$ is above a threshold, then for all $\rho$ and $\delta$ values, pay-per-use licensing always yields a higher consumer surplus (if $\beta>1$ ) and a higher social welfare (if $\beta>1.5$ ) than perpetual licensing. ${ }^{11}$

An interesting result in Proposition 4 is shown in Figure 3 (a). Under pure perpetual licensing, the optimal price is independent of the potential piracy $(\rho)$ when there is no network effect $(\beta=0)$, but when $\beta>0$, the optimal price increases with $\rho$. This is because higher piracy increases the network size allowing the vendor to charge legitimate users higher prices. However, the overall effect (shown in Figure 3 (b)) of higher piracy is lower vendor profits (i.e., the vendor's profit decreases with $\rho$ ). Our result

\footnotetext{
${ }^{11}$ Our consumer surplus and social welfare calculations under perpetual licensing include only legitimate users not pirated software users. Including illegal users in such calculations leads to "misleading" results-for example, one will then conclude that perpetual licensing always gives higher consumer surplus if the piracy rate is high enough.
} 
contrasts the finding of others (Conner and Rumelt 1991, Shy and Thisse 1999) that, in a market with network effects, allowing piracy can lead to higher firm profits than preventing it. This difference arises because Conner and Rumelt's model assumes an ad-hoc, linear aggregate demand function, whose slope is indirectly related to piracy and can be reduced by the firm's level of IP prevention technology, thus the total potential market size (from legitimate and pirate users) in their model is actually not fixed. We model a monopoly market whereas Shy and Thisse consider competition between two vendors, both of whom have incentives to achieve a higher network effect for their own product.
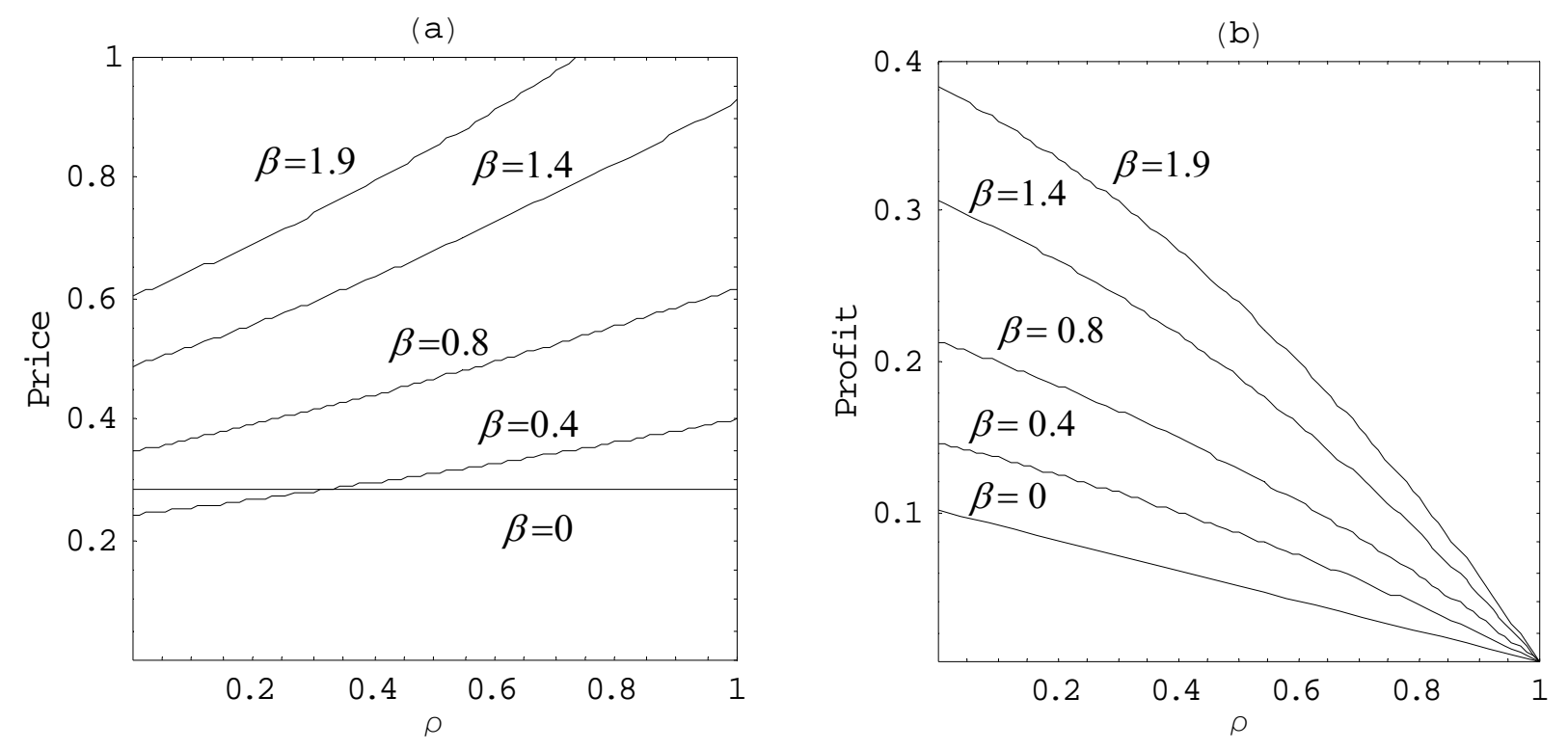

Figure 3: Optimal price and profit under perpetual licensing for different $\beta$ values.

Proposition 4 also states that if the network strength is weak and the potential piracy is relatively low (e.g., $\beta=0.4$ and $\rho=0.1$ in Figure 3 (a)), the vendor will have an incentive to lower its perpetual licensing price (from the level that it would charge under no network effects); otherwise, the optimal perpetual licensing price will be strictly higher with network effects than without. Intuitively, if the network effect is not strong, the vendor will find it more profitable to lower its price to increase its customer base rather than to increase its price to directly reap the benefits of network effect. 


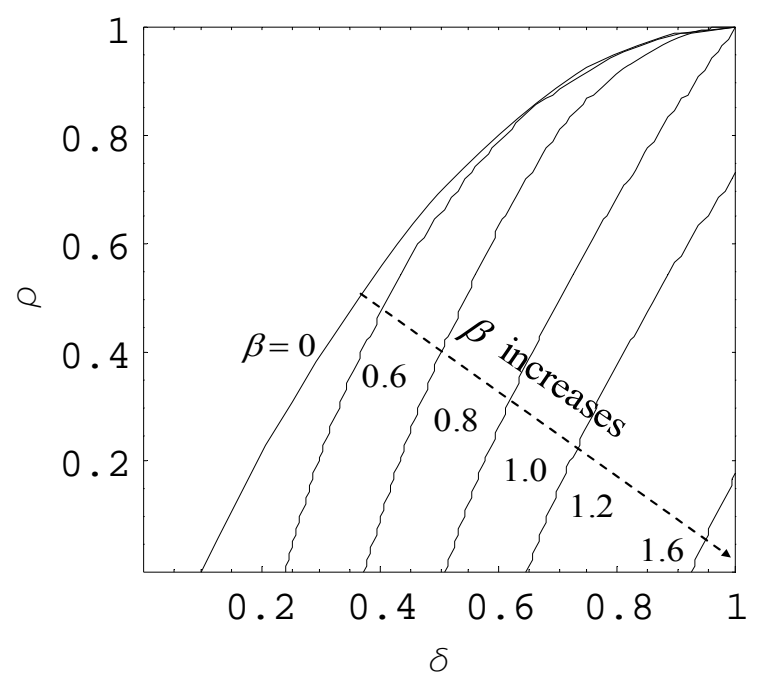

Figure 4: Profit cutoff curves in the $(\delta, \rho)$ plane shifts downward as $\beta$ increases; pay-per-use is more profitable than perpetual licensing only if $(\delta, \rho)$ lies above the cutoff curve.

Proposition 5 is illustrated in Figure 4. With a linear network effect, pay-per-use licensing is more profitable than perpetual licensing as long as $\delta$ is relatively low. More interestingly, the presence of network effects makes the benefit of pay-per-use licensing stronger. If $(\delta, \rho)$ lies above the cutoff curve (for a given $\beta$ ), pay-per-use is more profitable than perpetual licensing; if $(\delta, \rho)$ is below the cutoff curve, perpetual licensing is more profitable. The cutoff curve shifts downward as $\beta$ increases, i.e., the parameter region in which pay-per-use is more profitable than perpetual licensing becomes larger as $\beta$ increases. ${ }^{12}$ If $\beta>1.8$, pay-per-use will always be more profitable than perpetual licensing regardless of the user inconvenience cost $(\delta)$ or the potential piracy rate $(\rho)$. We find that the profit ratio between pay-per-use and perpetual licensing (for given $\delta, \rho$ values) increases with $\beta$. Similarly, Figure 5 shows that the cutoff curves, above which pay-per-use licensing leads to a higher consumer surplus or higher social welfare, also shift downwards as $\beta$ increases; that is, from an overall consumers' or societal point of view, the presence of network effect also makes pay-per-use licensing more favorable.

\footnotetext{
${ }^{12}$ The topmost cutoff curve in Figure 4 corresponds to the case of $\beta=0$, which is identical to what is expected from our analytical result in Proposition 1; this special case serves to verify the correctness of our numerical procedure for the general case of $\beta \geq 0$.
} 

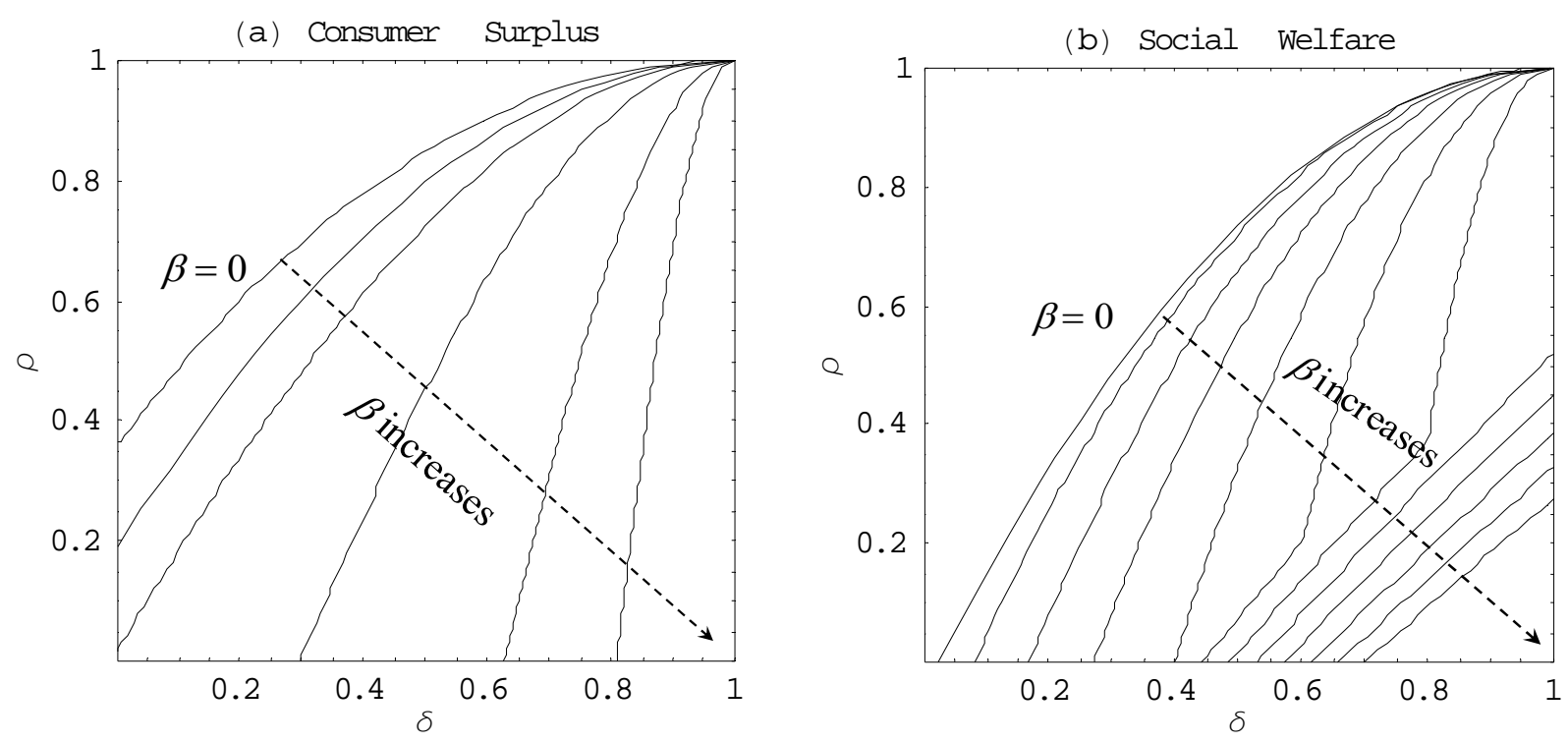

Figure 5: CS and SW cutoff curves in the $(\delta, \rho)$ plane shifts downward as $\beta$ increases; pay-per-use yields a higher CS (SW) than perpetual licensing only if $(\delta, \rho)$ lies above the curve.

\section{Licensing Of General Software (Model Robustness Check)}

We next extend our model to examine non-utility software. Thus far, we have analyzed licensing schemes assuming that each consumer has a constant marginal usage benefit and an exogenous usage level. However, for many applications, consumers may have decreasing marginal benefits; i.e., an individual's usage level may be determined by a decreasing marginal benefit function and the cost for additional usage. ${ }^{13}$ To model such consumer preferences, we adopt a linearly decreasing marginal benefit function: $\mu=a-\frac{\theta}{b}+\beta N$, where $a-\frac{\theta}{b}$ represents the intrinsic component of the marginal benefit (without consideration for any network effect), and $\beta N$ represents the component of the marginal benefit due to the network effect. Suppose that consumers are homogeneous in terms of the initial intrinsic marginal benefit ( $a$ ) and its sustainability $(b)$, which represents how slowly the intrinsic marginal benefit decreases with higher levels of software use. As before, we assume that a fraction $\rho$ of consumers have low acquisition costs $\left(\gamma_{l}\right)$ for pirated perpetual software while the rest has high acquisitions costs $\left(\gamma_{h}\right)$.

\footnotetext{
${ }^{13}$ There may also be some initial learning effect — marginal benefit may increase as users learn the software features. We leave it to future research to study such learning effects.
} 
The optimal profits for pay-per-use and perpetual licensing are easily found: $\Pi_{p p u}^{\max }=\frac{b(a+\beta-\delta)^{2}}{4}$, $\Pi_{p}^{\max }=\frac{b(1-\rho)(a+\beta)^{2}}{2}$. Hence, if $\rho<1-\frac{1}{2}\left(1-\frac{\delta}{a+\beta}\right)^{2}$, perpetual licensing is more profitable than pay-per-use licensing. That is, unless piracy is very high (higher than 50\%), even if the inconvenience cost of pay-per-use $(\delta)$ is zero, perpetual licensing is the optimal licensing choice when consumers have homogeneous valuations. We will see that in contrast, with heterogeneous consumers, pay-per-use licensing tends to be more optimal.

Now suppose that consumers are heterogeneous in terms of their marginal benefit. We assume that both $a$ and $b$ are uniformly distributed (and normalized): $a \sim$ uniform $[0,1]$ and $b \sim$ uniform $[0,1]$. Figure 6 shows four examples of user (intrinsic) marginal benefit curves - two with large $a$ values and two with small $a$ values, each of which has a different $b$ value.

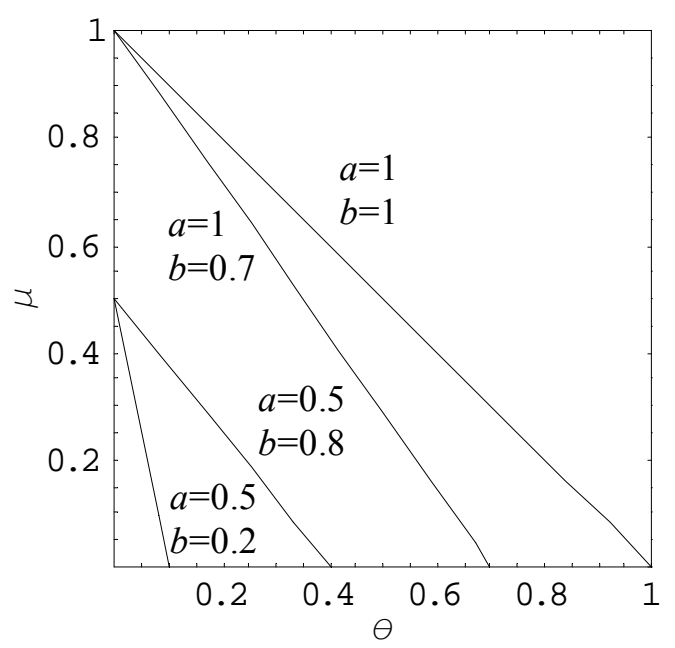

Figure 6: Examples of marginal benefit curves

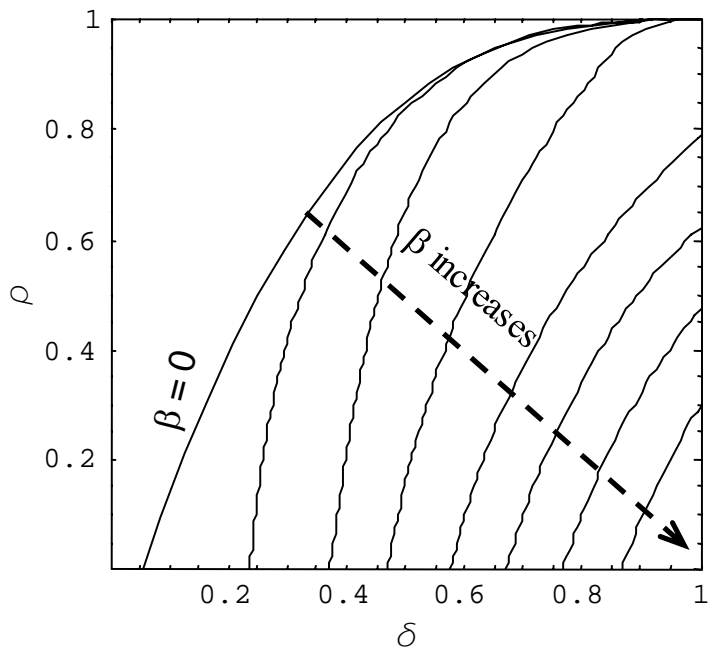

Figure 7: Profit cutoff curves in $(\delta, \rho)$ plane

The current model for the marginal benefit and consumer heterogeneity addresses two weaknesses in the utility software models of the previous sections. First, we now consider the correlation between a user's marginal benefit and the usage level. In particular, a consumer's marginal usage benefit decreases as his or her usage level increases. This is more general and more common than our model for utility software. Second, our current model also has a built-in characteristic that heavy users are much 
more likely to have a higher marginal benefit than light users. ${ }^{14}$ We will demonstrate that the results of our current analysis are qualitatively the same as those found in previous sections indicating that our main results are robust with respect to different assumptions about consumers' marginal benefits, software use behaviors, and consumer heterogeneity.

Under pay-per-use, a consumer will choose a level of usage such that her marginal benefit equals her marginal cost: $\mu=a-\frac{\theta}{b}+\beta N=P_{\theta}+\delta$. The vendor's profit is given by (1), and the fulfilled expectation equilibrium provides a simple identity (2) for the total number of users.

$$
\begin{aligned}
& \Pi_{p p u}=\iint P_{\theta} \theta d a d b=\int_{0}^{1} \int_{\max \left[0, P_{\theta}+\delta-\beta N\right]}^{1} P_{\theta} b\left(a+\beta N-P_{\theta}-\delta\right) d a d b \\
& N \equiv \int_{0}^{1} \int_{\max \left[0, P_{\theta}+\delta-\beta N\right]}^{1} d a d b
\end{aligned}
$$

We consider two cases (based on $P_{\theta}$ ) for the lower integration bound in (1) and (2), and then compare the maximum profits under these cases to find the optimal price and profit, which are given in (3).

$$
\left\{\begin{array}{l}
\Pi_{p p u}^{\max }=\frac{(1-\delta)^{3}}{27(1-\beta)^{2}}, P_{\theta}^{*}=\frac{1-\delta}{3}, \text { if } \beta \leq \frac{1+2 \delta}{3}, \\
\Pi_{p p u}^{\max }=\frac{\beta-\delta}{4}, P_{\theta}^{*}=\beta-\delta, \text { if } \frac{1+2 \delta}{3} \leq \beta \leq \frac{1}{2}+\delta, \\
\Pi_{p p u}^{\max }=\frac{(1+\beta-\delta)^{2}}{8}, P_{\theta}^{*}=\frac{1}{4}+\frac{\beta-\delta}{2}, \text { if } \beta \geq \frac{1}{2}+\delta .
\end{array}\right.
$$

Under perpetual licensing, once a consumer purchases the perpetual software, she will use the software until her marginal benefit equals her marginal cost (which is zero): $a-\frac{\theta_{\max }}{b}+\beta N=0$. Thus, a consumer of type $(a, b)$ with high acquisition costs for pirated software will buy the perpetual software if (4) holds: Total Benefit $=\int_{0}^{\theta_{\max }}\left(a-\frac{\theta}{b}+\beta N\right) d \theta=\frac{b(a+\beta N)^{2}}{2} \geq P$,

where the total number of users $N=N_{h}+\rho$. The fulfilled expectation equilibrium condition (5)

\footnotetext{
${ }^{14}$ It is straightforward to analytically show that, in our current model, the probability that heavy users have high marginal benefits is higher than the probability that light users have high marginal benefits.
} 
involves the integration region given by (4), which needs to be considered in two cases depending on whether the $b$-intercept is greater than unity.

$$
N_{h} \equiv(1-\rho) \iint_{(14)} d a d b=\left\{\begin{array}{l}
(1-\rho)\left(1-\frac{2 P}{\beta N(1+\beta N)}\right), \text { if } \frac{2 P}{(\beta N)^{2}} \leq 1 \\
(1-\rho)\left(\sqrt{1+\beta N}-\sqrt{\frac{2 P}{1+\beta N}}\right)^{2}, \text { if } \frac{2 P}{(\beta N)^{2}} \geq 1
\end{array}\right.
$$

From (5) and under each case, we can easily obtain an expression for $P$ in terms of $N_{h}$, and therefore

express the vendor's profit $\left(\Pi_{p}^{\max }=N_{h} P\right)$ in terms of only $N_{h}, \beta$ and $\rho$. We must, however, resort to numerical techniques again since closed form analytical solutions do not exist.

Our numerical analysis shows that the current model yields qualitatively the same results as those in section 5. A monopolist vendor will find pay-per-use licensing more favorable and more likely to dominate perpetual licensing as the network strength increases; if the network strength is above a threshold, pay-per-use is always more profitable than perpetual licensing, as depicted in Figure 7. The cutoff curves also resemble those found in section 5. The quantitative difference is that the $\beta$ threshold (above which pay-per-use is always preferred) is a little larger than that found earlier. Compared with the profit cutoff curves in earlier models (illustrated in Figure 4), for the same $\beta$ values, the corresponding current cutoff curve (in Figure 7) generally lies to the upper left. That is, if consumers are heterogeneous and have decreasing marginal benefits, the region of parameters $(\delta, \rho)$ for which pay-per-use yields higher profits than perpetual licensing is smaller than that when marginal benefits are constant. Thus, our conclusions in section 5, which are based on fixed individual usage levels and constant individual marginal benefits, are in fact qualitatively robust even in our current more general framework.

\section{Managerial Implications and Conclusions}

We have provided an analytical framework to study a software vendor's optimal licensing choice in a market with potential piracy, network effects, and customer heterogeneity. We find that consumer heterogeneity, the potential piracy rate, the user inconvenience cost of pay-per-use licensing, and the 
strength of the network effect are important factors to consider for a vendor's optimal choice of licensing architecture. With higher potential piracy, lower inconvenience costs, and stronger network effects, payper-use licensing becomes not only more profitable but also more welfare-enhancing. We find that, in a market without network effects, pure pay-per-use licensing yields higher profits than perpetual licensing or mixed licensing if the user inconvenience cost is low or if the potential piracy is relatively high. If the inconvenience cost is very high, however, a vendor is better off offering mixed licensing because adding a high-priced pay-per-use offering to a perpetual licensing option can give the vendor a net gain from the high valuation, light user segment (region R3 in Figure 2) even though the vendor forgoes some profits from users in region R4. We find that, with a linear network effect, the vendor's optimal perpetual licensing price increases with the piracy rate though its profit decreases with piracy. If the network effect is weak, a vendor offering perpetual software in a market with low piracy will find it optimal to lower its price from what it would charge under no network effect. Our analysis shows that in a market with heterogeneous consumers, the presence of network effect favors pay-per-use over perpetual licensing.

Our research has important managerial implications. Pay-per-use licensing helps firms better cope with very heterogeneous consumers in terms of valuation and usage. Managers of independent software vendors face a tradeoff between piracy prevention and licensing transparency or application user friendliness/convenience. The usage-based licensing architecture is an effective technology-based protection against software piracy and can lead to much higher profits than perpetual licensing if the user inconvenience cost is relatively low or if the network effect is strong. While software piracy will decrease as more vendors adopt DRM technologies, the barrier to adopting pay-per-use licensing (e.g., user inconvenience costs) also decreases significantly as the Internet becomes easier and cheaper to access and as the application interface design improves. The optimal licensing choice depends on the level of potential piracy, the user inconvenience cost, and the strength of the network effect (Figures 4 and 7). As the inconvenience cost becomes negligible, pay-per-use licensing is always preferred regardless of the piracy rate. Managers may find it optimal, if possible, to vary the licensing schemes across markets that have different potential piracy, user inconvenience costs, and/or network strength. For example, if digital 
piracy is very prevalent in some countries, it may be optimal for vendors to offer software localization with pay-per-use licensing in those markets. Unfortunately, inconvenience costs may also be high in those countries. Managers may estimate inconvenience costs and network strength through marketing research, and then use the IDC piracy rate estimates to refer to Figures 4 and 7 as a guideline to determine which licensing architecture is optimal.

Software vendors as well as legitimate users can benefit from piracy prevention through usagebased licensing. Through higher market penetration, vendors can capture more economic value added. In turn, they can offer better products and services to users, who will benefit from higher quality or variety and potentially lower prices. Vendors can also collect software usage information to help them focus their resources on developing and fixing software features that users need most and to potentially offer welltargeted promotions on new products or features, benefiting both vendors and users.

While digital piracy remains rampant, perpetual licensing is still prevalent in the software industry. Our analyses suggest some possible reasons: high user inconvenience cost for usage-based licensing, resistance from heavy users, or other factors such as the lack of consumer familiarity about this new licensing mechanism. The general business environment has evolved to a lower user inconvenience cost. Internet access and broadband network connections have gradually become more universal even in households. Financial transactions over the Internet have become cheaper and more secure. As web technology improves, it is likely that the inconvenience from pay-per-use will gradually become negligible, and that usage-based licensing such as pay-per-use or SaaS which offers technology-based IP protection can become easier and cheaper to use or support than traditional perpetual licensing.

Our future empirical work will investigate the effects of licensing architecture on piracy prevention and market penetration. Analytical and empirical studies on adoption and diffusion of DRM systems such as the licensing technologies provided by Agilis and Macrovision may be of both academic and practical interest. We expect that vendors facing the highest piracy losses and lowest inconvenience costs in a market with a high degree of user heterogeneity and strong network effects will be the early adopters of such licensing technologies. Our analysis has considered only linear network effects and 
rational expectation equilibria; a competitive and dynamic model with more realistic network effect may bring new insights. We hope our research motivates others to investigate these important questions.

\section{Appendix}

\section{Proof of Proposition 1:}

Suppose that the vendor offers only perpetual licensing. Users of type $\gamma_{l}$ derive a higher net utility using the pirated software than buying the legitimate software, and will thus not purchase any license. Thus, a fraction $\rho$ of all consumers (those of type $\gamma_{l}$ ) will use the pirated software when available. Type $\gamma_{h}$ consumers with $\mu \theta \geq P$ will purchase the perpetual software. The total number of high-type $\left(\gamma_{h}\right)$ consumers who will buy the perpetual software can easily be written as a function of price: $N_{h}=(1-\rho) \iint_{\mu \theta \geq P} d \mu d \theta=(1-P+P \ln P)(1-\rho)$, where $P \in(0,1]$. This is the aggregate demand function under perpetual licensing. The vendor's profit is given by $\Pi_{p}(P)=P(1-P+P \ln P)(1-\rho)$.

Profit maximization leads to $\Pi_{p}^{\max }=\Pi_{p}(0.285)=0.102(1-\rho)$ with a market share of $m=N_{h}=(1-P+P \ln P)(1-\rho)=0.358(1-\rho)$. The total consumer surplus (from legitimate users) is computed as $C S_{p}=\iint_{\mu \theta \geq 0.28467}(\mu \theta-0.285)(1-\rho) d \mu d \theta=0.077(1-\rho)$, while the social surplus is given by $S W_{p}=C S_{p}+\Pi_{p}^{\max }=0.179(1-\rho)$.

If the vendor offers only pay-per-use, all consumers with $\mu \geq P_{\theta}+\delta$ will pay to use the software. The aggregate demand under pay-per-use is given by $Q=\int_{0}^{1} \int_{P_{\theta}+\delta}^{1} \theta d \mu d \theta=\frac{(1-\delta)-P_{\theta}}{2}$. The vendor's profit function is thus given by $\Pi_{p p u}\left(P_{\theta}\right)=Q P_{\theta}=\frac{P_{\theta}(1-\delta)-P_{\theta}^{2}}{2}$, whose maximum is easily found to be $\Pi_{p p u}^{\max }=\Pi_{p p u}\left(\frac{1-\delta}{2}\right)=\frac{(1-\delta)^{2}}{8}$, resulting in a market share of $m=\frac{1-\delta}{2}$.

Comparing the optimal profits under these two licensing schemes, we easily find that the vendor will make a higher profit under pay-per-use if $\rho>1-1.228(1-\delta)^{2}$, and a higher profit under perpetual 
licensing if $\rho<1-1.228(1-\delta)^{2}$. Comparing the vendor's market shares under the two licensing schemes, we find that pay-per-use leads to a higher market share if and only if $\delta<0.285+0.715 \rho$.

\section{Proof of Proposition 2:}

Suppose that the vendor offers mixed licensing (i.e., both pay-per-use and perpetual licensing). If it sets $P \geq P_{\theta}+\delta$, no consumers will purchase the perpetual software because they can derive higher net utility using pay-per-use. Hence, if $P \geq P_{\theta}+\delta$, mixed licensing will yield a lower profit than pure payper-use because the presence of perpetual licensing removes the anti-piracy effect of pay-per-use without making any contribution to profits (due to overpricing). Hence, a profit-maximizing vendor will set $P \leq P_{\theta}+\delta$. Figure 2 depicts the case of $P \leq P_{\theta}+\delta$. For light users with $\theta<\frac{P}{P_{\theta}+\delta}$, pay-per-use has lower effective costs; for heavy users with $\theta \geq \frac{P}{P_{\theta}+\delta}$, perpetual licensing is preferable. Hence, the vendor's profit is given by (A1).

$$
\begin{aligned}
& \Pi_{\text {mixed }}\left(P, P_{\theta} ; \delta, \rho\right)=\left(\int_{P_{\theta}+\delta}^{1} \int_{0}^{\frac{P}{P_{\theta}+\delta}} P_{\theta} \theta d \theta d \mu+\int_{\frac{P}{P_{\theta}+\delta}}^{1} \int_{\frac{P}{\theta}}^{1} P d \mu d \theta\right)(1-\rho) \\
& =(1-\rho)\left(\frac{P^{2} P_{\theta}\left(1-\delta-P_{\theta}\right)}{2\left(P_{\theta}+\delta\right)^{2}}+P-\frac{P^{2}}{P_{\theta}+\delta}+P^{2} \ln \left(\frac{P}{P_{\theta}+\delta}\right)\right)
\end{aligned}
$$

We solve the optimization problem: $\max _{P, P_{\theta}} \Pi_{\text {mixed }}\left(P, P_{\theta} ; \delta, \rho\right)$, subject to the constraint $P-P_{\theta}-\delta \leq 0$.

Define the Lagrangian as $L=\Pi_{\text {mixed }}\left(P, P_{\theta} ; \delta, \rho\right)-\lambda\left(P-P_{\theta}-\delta\right)$. The first-order conditions can be simplified to the following:

$$
\begin{aligned}
& \frac{\partial L}{\partial P}=(1-\rho)\left(\frac{P P_{\theta}\left(1-\delta-P_{\theta}\right)}{\left(P_{\theta}+\delta\right)^{2}}+1-\frac{2 P}{P_{\theta}+\delta}+P+2 P \ln \left(\frac{P}{P_{\theta}+\delta}\right)\right)-\lambda=0 \\
& \frac{\partial L}{\partial P_{\theta}}=(1-\rho) P^{2}\left(\frac{1-\delta}{2\left(P_{\theta}+\delta\right)^{2}}+\frac{\delta}{\left(P_{\theta}+\delta\right)^{3}}-\frac{1}{P_{\theta}+\delta}\right)-\lambda=0
\end{aligned}
$$


Clearly, $\lambda=0$, because from earlier discussions the optimization constraint is non-binding. We can solve (A3) to obtain a unique non-negative solution $P_{\theta}^{*}=\frac{1-5 \delta+\sqrt{(1+\delta)^{2}+12 \delta}}{4}$. Substituting this solution into (A2), we can then solve for $P^{*}$ in terms of $\delta$. Unfortunately, (A2) does not have a closed form solution. However, we can readily obtain $P^{*}$ numerically for any given $\delta$. We then verify that the solution pair $\left(P_{\theta}^{*}, P^{*}\right)$ satisfies the constraint $P-P_{\theta}-\delta \leq 0$. Our numerical study shows that for each $\delta \in[0,1]$, there exists a unique maximum $\left(P_{\theta}^{*}, P^{*}\right)$ that satisfies the optimization constraint. It is easy to prove analytically that $\Pi_{\text {mixed }}\left(P, P_{\theta} ; \delta, \rho\right)$ is a monotonically decreasing function in $\delta$, a fact that is also intuitively clear because all else being equal, reducing the inconvenience cost implies that the vendor will benefit by increasing its pay-per-use price. Thus, the maximum profit under mixed licensing, denoted by $\Pi{ }_{\text {mixed }}^{\max }(\delta, \rho)$, is a decreasing function in $\delta$, and it is straightforward to show that $\Pi_{\text {mixed }}^{\max }(\delta, \rho) \leq \frac{1-\rho}{8}$ by setting $\delta=0$ to solve for $\left(P_{\theta}^{*}, P^{*}\right)$.

Note that with fixed costs sunk, adding pay-per-use licensing will improve the vendor's profit if $P_{\theta}$ is set high enough; i.e., mixed licensing is more profitable than perpetual licensing. From our analysis of Proposition 1, pure pay-per-use licensing yields an optimal profit of $\Pi_{p p u}^{\max }=\frac{(1-\delta)^{2}}{8}$ and pure perpetual licensing yields $\Pi_{p}^{\max }=0.10182(1-\rho)$. Proposition 2 follows readily by comparing the profits under each licensing scenario.

\section{Analysis of Perpetual Licensing of Utility Software in a Market with Linear Network Effect:}

Note that type- $\gamma_{l}$ consumers will use the pirated perpetual software and type- $\gamma_{h}$ consumers will purchase the perpetual software only if $\mu \theta-P \geq 0$. With the linear network effect of the form $\mu=\mu_{0}+\beta N$, we can rewrite the purchasing condition as $\mu_{0} \geq \frac{P}{\theta}-\beta N$, where the total number of users 
includes some type- $\gamma_{h}$ users and all type- $\gamma_{l}$ users (i.e., $N=N_{h}+\rho$ ). Figure A-1 illustrates two possible pricing scenarios depending on whether (a) $P \leq \beta N$ or (b) $P>\beta N$.

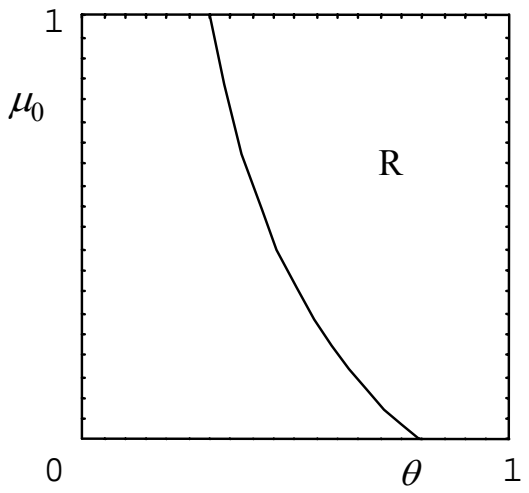

(a)

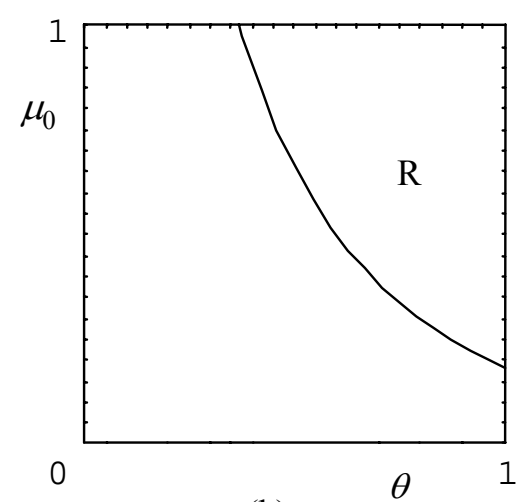

(b)

Figure A-1: Two cases for the integration region R: $\mu_{0} \geq \frac{P}{\theta}-\beta N$

The total number of consumers purchasing the perpetual software is represented by area R:

$$
N_{h}=(1-\rho) \int_{\text {area } R} d \theta d \mu
$$

The vendor's profit is given by $\Pi_{p}=P \cdot N_{h}$

In case (a), simplification of (A4) and (A5) leads to

$$
\Pi_{p}\left(N_{h}\right)=\frac{N_{h}-\frac{N_{h}^{2}}{1-\rho}}{\ln \left[1+\frac{1}{\beta\left(N_{h}+\rho\right)}\right]},
$$

which can be optimized by solving the first order condition (FOC) for $N_{h}$, from which the optimal price $P$ can be analytically calculated. The consumer surplus in case (a) is given by (A7).

$C S_{p}^{(a)}=(1-\rho)\left[\int_{P /(1+\beta N)}^{P / \beta N} \int_{\frac{P}{\theta}-\beta N}^{1}\left(\left(\mu_{0}+\beta N\right) \theta-P\right) d \mu_{0} d \theta+\int_{P / \beta N}^{1} \int_{0}^{1}\left(\left(\mu_{0}+\beta N\right) \theta-P\right) d \mu_{0} d \theta\right]$

Case (b) is more complicated because equation (A4) simplifies to (A8), which cannot be manipulated into a separable form. 


$$
N_{h}=(1-\rho)\left[1+\beta\left(N_{h}+\rho\right)-P+P \cdot \ln \left(\frac{P}{1+\beta\left(N_{h}+\rho\right)}\right)\right]
$$

The FOC for profit maximization can be simplified by implicitly differentiating (A5) and (A8) to give

$$
\frac{d \Pi_{p}}{d N_{h}}=P+N_{h} \frac{d P}{d N_{h}}=P+\frac{N_{h}\left(\frac{1}{1-\rho}+\frac{\beta P}{1+\beta\left(N_{h}+\rho\right)}-\beta\right)}{\ln \left(\frac{P}{1+\beta\left(N_{h}+\rho\right)}\right)}=0
$$

The consumer surplus in case (b) is given by $C S_{p}^{(b)}=(1-\rho) \int_{\frac{P}{1+\beta N}}^{1} \int_{\frac{P}{\theta}-\beta N}^{1}\left(\left(\mu_{0}+\beta N\right) \theta-P\right) d \mu_{0} d \theta$

We can then solve (A8) and (A9) simultaneously for the optimal $N_{h}$ and $P$. Clearly, no closed form solutions exist for either case (a) or (b). We resort to a complete numerical analysis for solutions. After finding the optima from FOC, we must verify whether the constraints for each case are satisfied accordingly, and then compare the optima with the boundary values to find the unique global maximum profit. For each combination in a 200x200 grid of $(\rho, \beta)$ values, we compute the optimal price, profit, market share, consumer surplus, and social welfare.

\section{References}

August, T. W., Tunca, T. 2007. Let the Pirates Patch? An Economic Analysis of Network Software Security Patch Restrictions. Information Systems Research, Forthcoming.

Bakos, Y., E.Brynjolfsson. 1999. Bundling information goods: Pricing, profits, and efficiency. Management Science 45(12) 1613-1631.

Chen, Y., I. Png. 2003. Information goods pricing and copyright enforcement: Welfare analysis. Information Systems Research 14(1) 107-123.

Choudhary V., K. Tomak, A. Chaturvedi. 1998. Economic Benefits of Renting Software. Journal of Organizational Computing and Electronic Commerce 8(4) 277-305.

Conner, K. R., R. P. Rumelt. 1991. Software Piracy: An Analysis of Protection Strategies. Management Science 37(2) 125-139. 
Dusparic, I. and J. Dowling. 2003. Mobile Software Licensing. Technical Report, the University of Dublin. https://www.cs.tcd.ie/publications/tech-reports/reports.03/TCD-CS-2003-29.pdf

Essegaier, S., S. Gupta, Z.J. Zhang. 2002. Pricing Access Services. Marketing Science 21(2) 139-159.

Gurnani, H., K. Karlapalem. 2001. Optimal Pricing Strategies For Internet-Based Software

Dissemination. The Journal of the Operational Research Society 52 64-70.

International Data Corporation. 2005. Second Annual BSA and IDC Global Software Piracy Study, May 2005. http://www.bsa.org/globalstudy/upload/2005-Global-Study-English.pdf

Jiang, B., P. Chen, T. Mukhopadhyay. 2007. On-demand enterprise software: the software as a service model. Tepper School of Business Working Paper, Carnegie Mellon University.

Jiang, B. 2007. An economic analysis of ad-supported software. Tepper School of Business Working Paper, Carnegie Mellon University.

Katz, Michael L., Carl Shapiro. 1985. Network externalities, competition, and compatibility. American Economic Review 75(3) 424-440.

Konary, A., S. Graham, L. Seymour. 2004. "The future of software licensing: Software Licensing under Siege", White Paper, International Data Corporation.

Kulatilaka, N., L. Lin. 2006. Impact of Licensing on Investment and Financing of Technology Development. Management Science 52(12) 1824-1837.

Ma, D. and A. Seidmann. 2007. ASP On-Demand Versus MOTS In-House Software Solutions. Working Paper, available at SSRN: http://ssrn.com/abstract=996774.

Macrovision Corporation. Licensing Module: Price, Package and License Software Any Way Your Customers Want. Macrovision Product Literature.

Macrovision Corporation. Flexibly Price, Package, Protect and Update your Software Products. http://www.macrovision.com/pdfs/fnp brochure.pdf, accessed May, 2006.

Shapiro, C., H.Varian. 1998. Information Rules: A Strategic Guide for the Network Economy. Harvard Business School Press, Cambridge, MA. 
Sundararajan, A., 2004a. Managing Digital Piracy: Pricing and Protection. Information Systems Research 15 287-304.

Sundararajan, A. 2004b. Non-linear pricing of information goods. Management Science 50(12) 16601673.

Shy, O., J. F. Thisse. 1999. A Strategic Approach to Software Protection. Journal of Economics and Management Strategy 8(2) 163-190.

Takeyama, L. N. 1994. The Welfare Implications of Unauthorized Reproduction of Intellectual Property in the Presence of Network Externalities. Journal of Industrial Economics. 42(2) 155-166.

Thibodeau, P. 2005. New Reasons to Do It Yourself. Computerworld. 39(36) 38.

Wu, S. and P. Chen. 2005. Fighting Information Good Piracy with Versioning. Tepper School Working Paper, Carnegie Mellon University.

Zhang, J. and A. Seidmann. 2002. The Optimal Software Licensing Policy Under Quality Uncertainty. Proceedings of the 23rd International Conference on Information Systems, L. Applegate, R. D. Galliers, and J. I. DeGross (Eds.), Barcelona, 225-236. 